\title{
MODELOS UNIVERSITARIOS EN PUGNA: DEMOCRATIZACIÓN O MERCANTILIZACIÓN DE LA UNIVERSIDAD Y DEL CONOCIMIENTO PÚBLICO EN ARGENTINA
}

\author{
Fernanda Juarros ${ }^{1}$ \\ JUDITH NAIDORF ${ }^{2}$
}

Recebido em: 07/07/07

Avaliado em: 24/08/07

1 Docente investigadora y becaria doctoral de la Universidad de Buenos Aires.

E-mail: fjuarros@clacso.edu.ar

2 Docente de la Universidad de Buenos Aires. UBA-CONICET

Resumen: Los cambios ocurridos en el sistema universitario argentino se vinculan al modelo económico neoliberal que cobró fuerza en los 90. A partir de entonces, las universidades comenzaron a adecuar sus actividades a una nueva cultura de interacción con el sector productivo en busca de competitividad.

En este escenario ubicamos dos discursos en disputa: uno enmarcado en las tendencias a democratizar los espacios públicos y el conocimiento libre; y otro vinculado a la tendencia de mercantilizar la educación y el conocimiento. Ambos tienen presencia en la universidad de hoy y su convivencia da lugar a posicionamientos fuertemente antagónicos que se corresponden con posiciones encontradas acerca de dos distintos modelos universitarios.

Palabras claves: democratización de la educación superior; mercantilización de la educación superior; modelos universitarios.

University models en pugna: Democratization or mercantilizacion of the university and of public knowledge in Argentina

Abstract: The changes that occurred in the Argentine university system are connected to the neoliberal economic model which was reinforced in the 90s. Since then, the universities adapted their activities to a new culture of interaction with the productive sector in search of competitiveness.

In this context, we locate two speeches: one affirms the trend of democratizing public spaces and free knowledge; and the other, the trend to commercialize knowledge. Both circulate in the university today in strongly antagonistic positions that correspond with two different university models.

Key words: Higher Education Democratization; Commercialization of higher education; university models. 


\section{INTRODUCCIÓN}

La democracia en Latinoamérica no se puede explicar desvinculada del proceso de liberación nacional de nuestros pueblos, por eso la Reforma Universitaria de 1918 que tuvo lugar en Argentina y se extendió a todos los países de la región, debe ser entendida como un movimiento político por encima de sus proyecciones pedagógicas. La Universidad no podía quedar al margen del impulso democratizador que estaba transitando la sociedad, e instaló en toda la región la lucha por democratizar el acceso a la educación superior, y movilizar a la sociedad para alcanzar la emancipación política de América Latina. Sin embargo, algunos de sus principios como la autonomía, el cogobierno, la libertad de cátedra y la extensión universitaria, parecerían comenzar a desdibujarse en el nuevo escenario que se viene configurando desde fin del siglo XX en el contexto de la región.

Los años 80 representan una clara ruptura, no sólo en materia educativa sino también en términos de política económica y social. De hecho, los cambios ocurridos en el sistema universitario argentino no pueden desvincularse del modelo económico neoliberal que comenzó a implementarse en los 90 a partir de políticas de privatización de empresas públicas, imposición de aranceles en el financiamiento de servicios públicos, medidas éstas asistidas por un discurso hegemónico sustentado en la competitividad económica internacional. Como parte del giro ideológico que tuvo lugar en esos años puede percibirse una nueva tendencia en el discurso y en las políticas de gobierno sobre Educación Superior que rompe con la tradición reformista establecida durante décadas anteriores.

En este nuevo escenario, las universidades recibieron el mandato de fortalecer la "excelencia" en docencia e investigación, y adecuar la calidad de sus actividades en una nueva cultura de interacción con el sector productivo en busca de competitividad. A partir de entonces, la universidad ha devenido en sujeto de distintos sectores que actúan por fuera de ella y que condicionan tanto el mantenimiento de sus estructuras organizativas y como su producción de conocimiento. Se ve entonces interpelada por múltiples requerimientos del Estado, de las empresas, de sus propios actores. En este sentido, uno de los conflictos a resolver por parte de los gobiernos de la región y de las propias universidades es cómo lograr la concertación de esos sectores: el sector público, el sector privado y la comunidad académica; y es en las di- 
ferentes concepciones acerca de cómo dirimir dicho conflicto donde se ponen en juego dos discursos en pugna que abogan, ya sea, por democratizar los espacios públicos y el conocimiento libre y para el bien común o una tendencia que denominamos, junto con muchos otros autores, de la mercantilización de la educación y el conocimiento. Ambos tienen su presencia en la universidad latinoamericana dando lugar a posicionamientos antagónicos respecto de sus políticas, e impactado tanto en las prácticas como en las formas de organización de cada institución, suscitando resultados distintos en cada una de ellas en función de las historias particulares que las determinan y fundamentalmente de su composición de fuerzas ${ }^{3}$.

La Universidad como toda institución demanda una permanente revisión tanto de sus planteamientos como de los mecanismos que determinan los dispositivos legales que le dan vida. El presente artículo no pretende ser exhaustivo en el análisis de las dimensiones que comprende el tema, intenta plantear algunas líneas de discusión y reflexión a partir de la necesidad de atender esta problemática que se impone con creciente preocupación en la agenda de la Educación Superior en América Latina.

\section{UN DEBATE TEÓRICO Y POLÍTICO: LOS MODELOS UNIVERSITARIOS EN PUGNA}

Durante las últimas décadas del siglo XX, el sistema de educación superior de la Argentina, al igual que el de muchos países de la región, debió afrontar la presión generada por el aumento de la población estudiantil y la disminución del financiamiento gubernamental. El proceso de resolución de esta tensión tuvo tres dimensiones: una dimensión técnica relacionada con la propuesta de alternativas, la generación de consensos y la toma de decisiones; una dimensión política relacionada con el poder relativo de los distintos actores involucrados para defender o atacar las diferentes opciones; y una dimensión filosófica relacionada con los propósitos últimos de la educación superior. En el centro del debate se encuentra una de las cuestiones más difíciles que deben enfrentar los gobiernos democráticos y las univer-

3 En Argentina la Universidad de Buenos Aires y la Universidad Nacional del Comahue han sido algunas de las que más se han resistido a dichos cambios. 
sidades: cómo armonizar los objetivos del sector público, los intereses privados y el ethos académico (LLOMOVATTE et al., 2006). Éste configura un debate teórico y político que ha recorrido la historia de la universidad en Argentina y que se actualiza en el marco de las controversias y deliberaciones a partir de la sanción de la Ley de Educación Superior (LEY 24.521) en el año $1995^{4}$.

En este escenario ubicamos dos discursos en disputa: uno que distintos autores coinciden en identificar las tendencias a democratizar los espacios públicos y el conocimiento libre y para el bien común y por otra parte la tendencia que denominamos, junto con muchos otros autores, de la mercantilización de la educación y el conocimiento. Ambos tienen su presencia en la universidad de hoy y su convivencia da lugar a posicionamientos fuertemente antagónicos que se corresponden con posiciones encontradas acerca de dos distintos modelos universitarios. Al respecto Martinetto (2007) plantea que detrás de la caracterización de la crisis actual de la universidad pública en Argentina, se pueden descubrir los elementos constitutivos de los posibles y diferentes modelos universitarios propuestos y en pugna por convertirse en el modelo hegemónico a instaurar. Estos distintos modelos son sostenidos por los decisores de la política pública, por el mercado, representado por empresarios, políticos, etc., por los actores ligados a la vida académica desde diferentes posiciones en la sociedad y por último, la sociedad como un todo, representada por las voces que nos llegan a través de distintos medios. Tan diversos actores, tienen intereses encontrados dentro de los cambios necesarios que la sociedad y la misma universidad está reclamando. Por lo tanto, el análisis de la crisis se verá teñido por estos mismos intereses que mueven a los distintos grupos a entrar en la lucha de poder por ganar espacios y lograr universalizar su visión de grupo como la única válida y lograr así imponer el modelo universitario propio como el ejemplo a seguir (MARTINETTO, 2007).

El discurso de la mercantilización de la educación y el conocimiento, ha impulsado proyectos de política universitaria centrados en el fortalecimiento de la calidad, en la diversificación de las fuentes de financiamiento y en la búsqueda de incremento de la competencia

4 Hasta 1995, la Argentina no tenía un instrumento normativo que regulara el funcionamiento de la educación superior en su conjunto. Las normas vigentes hasta entonces provenían de la agregación y en ocasiones de la superposición de leyes, decretos y resoluciones ministeriales diversas. 
entre las instituciones para mejorar eficiencia, entre otros. Esta posición ha generado en las universidades argentinas cambios que se evidencian en nuevas estructuras universitarias a fin de incrementar y multiplicar las fuentes externas de financiamiento -como las Unidades de Vinculación Tecnológica para facilitar la vinculación universidad-empresa así como la búsqueda denodada de los equipos de investigación por aplicar a subsidios que gestiona el Estado Nacional a través de la Agencia Nacional de Promoción Científica y Tecnológica provenientes del BID, entre otros -, implementación de fuertes mecanismos de control y deshomologación salarial de los docentes principalmente a través de programas de incentivos, proliferación de oferta de posgrados arancelados, las restricciones en el acceso y también cambios en la cultura académica que se definen más proclives que épocas anteriores a aceptar éstas reglas nuevas en la universidad (NAIDORF, 2005; SCHUGURENSKY; NAIDORF, 2004). Todo esto se enmarca de manera transparente en los lineamientos establecidos por el Banco Mundial.

Así, en el documento titulado "La Enseñanza Superior. Las lecciones derivadas de la experiencia" (1995), elaborado por el Departamento de Educación y Política Social del Banco Mundial, se sugieren las cuatro direcciones fundamentales para la reforma en educación superior: i) fomentar la mayor diferenciación de las instituciones, incluido el desarrollo de instituciones privadas; ii) proporcionar incentivos para que la instituciones públicas diversifiquen las fuentes de financiación; iii) redefinir la función del gobierno en la enseñanza superior; iv) adoptar políticas orientadas a otorgar prioridad a los objetivos de calidad y equidad (IRIARTE, 2004; RAMACCIOTTI, 2004). En un resumen ejecutivo del documento del Banco Mundial de julio de 1999, se puede leer: “¿Cuán productiva es la Educación Superior en América Latina? ¿Se podrían reasignar los recursos para mejorar la productividad?" Al respecto se responde: "la asignación de recursos dentro de la universidad pública latinoamericana es ineficiente [...] Para mejorar la eficiencia interna se precisará poder evaluar los costos y la productividad." (BANCO MUNDIAL, 1999, p. 26). Luego de diagnosticar los principales problemas de la educación superior relacionados con el gobierno, la administración y la productividad en Latinoamérica, se determina que: “...los gastos gubernamentales en Educación Superior se restringirán en un futuro próximo, y las instituciones de Educación Superior son 
excesivamente dependientes de fuentes únicas de ingresos" (p. 34). Se advierten como políticas alternativas el incremento de la participación privada en la financiación de la educación superior, junto con la estrecha correlación entre el presupuesto de la educación superior pública y el desempeño de la Universidad en el logro de objetivos del Gobierno. Ejerciendo estos lineamientos, tuvieron lugar en Argentina algunas experiencias en instituciones que lograron instituir el pago de matrícula, como en la Universidad Nacional de Córdoba, y también tuvieron lugar experiencias fallidas de instituciones que intentaron implantar la medida pero sin éxito, debido a los conflictos que desencadenaron ${ }^{5}$. De este modo, en la década del '90 y hasta nuestros días, podemos definir la introducción de un proyecto de política universitaria, que si bien fue resistido por buena parte de la comunidad universitaria, no pudo ser desarticulado, dado que también contó con defensores dentro de la misma.

En contraposición a lo planteado, el discurso de las políticas democratizadoras, en especial aquellas que coadyuvan a una mayor inserción social de las universidades públicas, intentan promover tipos de intervención que garanticen la plena vigencia de una ética tendiente a la construcción de una sociedad igualitaria en la que los saberes que se construyen y que se transmiten tengan ese papel transformador. Es preciso comenzar a debatir aspectos relevantes pendientes que demandan estas políticas universitarias como: i) el resguardo de la autonomía universitaria, ii) la defensa del acceso libre y gratuito al conocimiento, iii) la revisión de la agenda de la investigación académica, iv) la resignificación de la función de la extensión universitaria. Intentaremos a continuación profundizar los alcances de cada uno de ellos.

\section{i) El resguardo de la autonomía universitaria}

Existe consenso entre los especialistas acerca de las restricciones impuestas a la autonomía universitaria por parte de los gobiernos durante las décadas del 80, del 90 e incluso hoy en día. Esta intervención del Estado en la educación universitaria adopta diversas modalidades,

5 Éste fue el caso de la Universidad Nacional de la Patagonia Austral, donde se intentó imponer en el año 1999 el pago de un bono de \$10 mensuales por alumno, al que el claustro estudiantil se resistió. Asimismo, en las comisiones de trabajo por la reforma del estatuto de la Universidad de Buenos Aires (2007) se plantearon propuestas para establecer "cooperadoras" y aranceles aunque no prosperó en el consenso final. 
desde las más sutiles formas de presión gubernamental hasta las más evidentes manifestaciones de intromisión a través del establecimiento de reglas, imposición de normas y legislación, y esencialmente a través del manejo de las asignaciones presupuestarias.

El investigador canadiense, Ian Winchester (1985), sintetiza los tres argumentos más utilizados por los gobiernos para limitar la autonomía.

- En primer lugar, los gobiernos deben poder tomar decisiones acerca de política universitaria, dado que las fuentes de financiamiento siguen siendo predominantemente gubernamentales.

- En segundo lugar, las universidades han dejado de ser «torres de marfil»y son ahora parte integral de la sociedad que las cobija y, por lo tanto, el gobierno debe asumir responsabilidad directa de sus actividades, en tanto representante de la totalidad del cuerpo social. - En tercer lugar, dado que las universidades no son la única institución educativa terciaria y tampoco el único ámbito donde se realiza investigación, el gobierno debe coordinarlos a todos.

La conclusión a la que se arriba en ese trabajo sugerente es que el Estado no puede evitar ser un socio en la compleja tarea de las universidades (LLOMOVATTE, 2006).

Una mirada desde América Latina nos brinda una visión muy concreta de la nueva injerencia integral del Estado en la Universidad:

Dependiente en términos financieros del Estado, y en algunos casos de instituciones privadas, la universidad actual ha debido negociar sus propias definiciones frente al poder político y económico, en una lógica en la que se manifiesta la tensión entre los principios y las prácticas tradicionales de la universidad, frente a la racionalidad gubernamental y los principios del mercado.

Es decir, actualmente, la vida académica en las universidades argentinas se está viendo tan sujeta al control externo, que la autonomía que se requiere como componente necesario del profesionalismo se está perdiendo. Es fácil ver cómo se entrelazan y se potencian recíprocamente ambas acepciones del concepto de autonomía: por un lado, las nuevas pautas de evaluación externa a que están siendo sometidos los académicos y, por el otro, la necesidad de adaptación a las demandas de los diversos mercados que tienen cada vez mayor injerencia en la formación y en la investigación llevadas a cabo en las universidades. 
Para Pratt (1994),

las agresiones a la libertad académica provenientes de la controversia política y social siguen abundando, aunque sus formas cambian según la época. Las presiones económicas que se ejercen sobre nuestras instituciones no han cesado, y no dan señales de que esto vaya a ocurrir [...] En resumen, tanto la estabilidad como los estándares de nuestra profesión están bajo fuego ${ }^{6}$.

En Argentina, la universidad pública es una de las instituciones de mayor importancia desde el punto de vista del desarrollo científico; reúne el 54\% del personal dedicado a las actividades de I\&D en el país (BISANG, 1995) e históricamente ha recibido casi exclusivamente financiamiento estatal. Sin embargo, a partir de las transformaciones operadas, esta situación se ha modificado sustancialmente, obligándola a competir por subsidios que en décadas anteriores tenía garantizados por el Estado. La ley 24.521 es explícita al respecto: establece que es responsabilidad del Gobierno Nacional definir los roles del gobierno en materia de planeamiento, financiamiento y evaluación, e incorporar procesos de evaluación institucional interna y externa como herramientas para alcanzar el mejoramiento de la calidad y la modernización de las instituciones. Autoriza el cobro de colegiaturas en las entidades públicas, en contradicción con el principio de gratuidad de todos los niveles establecido en la Constitución Nacional, y establece un marco común para los sectores público y privado a través de la Comisión Nacional de Evaluación y Acreditación Universitaria (CONEAU), enmarcándose de este modo en las nuevas formas de intervención propias del Estado Evaluador ${ }^{7}$.

\footnotetext{
6 Pratt (1994). «The assaults on academic freedom arising out of political and social controversy continue to abound, though their forms change from season to season. The economic pressures on our institutions have not let up and show no signs of doing so [...] In short, both the stability and the standards of our profession are under fire.»

7 El Estado Evaluador es una racionalización y una redistribución general de funciones entre el centro y la periferia, de manera tal que el centro conserva el control estratégico global, por medio de palancas políticas menores en número, pero más precisas, constituidas por la asignación de misiones. Es el clásico quiebre entre pensamiento y acción, cuando se decide en la cúspide y se implanta en la base, dentro de una visión vertical, que se contrapone a formas democráticas de hacer donde los flujos directivos y los operacionales se encuentran en niveles medios, y la evaluación adopta otras características, como podrían ser los seguimientos longitudinales periódicos (PORTER, 2001).
} 
Estas medidas de política universitaria restringen la autonomía universitaria y redefiniendo la función y misión de la universidad. Esto es particularmente un llamado de alerta si entendemos la autonomía universitaria como la capacidad para definir tanto el contenido como los criterios de evaluación de lo que hace y produce (TENTI Fanfani, 1993) y consideramos que constituye una condición necesaria para el surgimiento y la difusión del auténtico conocimiento científico siendo, al mismo tiempo, el fundamento de la autoridad que los intelectuales comprometen en las luchas políticas.

\section{ii) El acceso a la Universidad como derecho al acceso al conocimiento}

En los últimos años el ingreso a la Universidad en Argentina, esencialmente en el ámbito público, aparece como un tema problemático. Las políticas de admisión que buscan regular el acceso a este nivel mediante diversos procedimientos, constituye un componente cada vez más controvertido en las políticas de educación superior. Estos procedimientos se supeditan de algún modo, a las condiciones contextuales que en función de la relación demandantes-poder público establecen acuerdos mínimos como los requisitos exigibles a los aspirantes. Allí se sitúa la discusión acerca de si estos requisitos exigibles se materializan en la sola acreditación de la aprobación del nivel medio o si estos requisitos exigibles se materializan en la superación de determinados dispositivos de selección. De este modo, este debate muchas veces queda reducido a estas dos posiciones antagónicas. Las mismas son definidas casi con sustento dogmático como aquella que propicia la "selectividad" y es equivalente a la propuesta del ingreso restringido por un lado, y aquella que argumenta la "apertura democrática" y se corresponde con la defensa del ingreso irrestricto y directo, por el otro.

En Argentina, la Ley de Educación Superior si bien mantiene la responsabilidad principal del Estado en el sustento de la educación superior pública universitaria, establece que para la distribución del aporte fiscal se tendrán en cuenta especialmente indicadores de eficiencia y equidad. En este marco, se establecen las bases normativas que posibilitan la limitación en el acceso, y la legislación argentina sobre estos temas han girado, con variantes, alrededor de los dos modelos señalados. Coexisten en las universidades públicas dos siste- 
mas de ingreso con sus correspondientes variantes. El ingreso directo puede o no ir acompañado de cursos introductorios, algunas veces incorporados a la currícula y otras, organizados por cada unidad académica con carácter no eliminatorio. Por otra parte, el ingreso restringido se obtiene a partir de la aprobación de un examen selectivo y puede ir o no acompañado de un cupo determinado por carrera (CHIROLEU, 1999).

La tendencia a promover el proceso selectivo se fundamenta en la inadecuación de las posibilidades de absorción de graduados por parte del mercado laboral, en el costo a la sociedad y en el presupuesto insuficiente a partir del cual la cantidad atentaría contra la calidad. La idea de aplicar mecanismos de racionamiento se basaría, de este modo, en el reconocimiento de la existencia de una cierta capacidad de recursos físicos y humanos, de manera que si esa capacidad no es respetada, el ajuste se daría por la vía de disminución de la calidad. La excelencia sólo podría estar asegurada en instituciones que utilicen mecanismos para certificar un ingreso anual regulado a partir de las disponibilidades físicas y los recursos humanos del establecimiento. En síntesis, la dificultad para mantener la calidad en contextos de masividad es el argumento más usado. Sin embargo, no podemos desconocer que fue la Universidad de Buenos Aires, universidad masiva que siguió al triunfo de la protesta estudiantil de 1918, quien produjo como fruto de su calidad tres premios Nobel de ciencia ${ }^{8}$.

Sabemos que por otro lado, la selección luego del ingreso se da a partir de los resultados académicos que los estudiantes obtienen esencialmente en los primeros años de sus carreras; resultados que reincidentemente se confrontan con las condiciones socioeconómicas de origen y muy frecuentemente con sus trayectorias educativas anteriores. Desde el punto de vista de la equidad, los estudios señalan que los beneficiarios de la educación universitaria se sitúan en los grupos de ingresos medios y altos aún en sistemas con ingreso libre y gratuito. Si bien en los últimos años se han implementado de manera creciente sistemas de ayuda económica para estudiantes de bajos recursos resultan todavía insuficiente para garantizar una mínima igualdad de oportunidades. 
Es verdad que una universidad en tensión con un sistema que subvierte los valores de una democracia sustantiva en favor de los valores del mercado no puede generar y distribuir el conocimiento de una manera justa, pero si puede decidir a quienes y entre quienes socializar esos saberes. La argumentación a favor del ingreso directo se basa en sostener que éste garantizaría la igualdad de oportunidades, la inclusión de los sectores menos favorecidos, el aumento de la equidad. Plantea que la educación superior cumpliría otras funciones no tradicionales como la socialización juvenil o la ampliación de la base cultural de la población, considerando la cantidad de ingresantes como factor de democratización de la educación superior.

\section{iii) La redefinición de la agenda de la investigación}

\section{académica}

Las perspectivas de análisis acerca de la producción de conocimiento difieren entre los países industrializados o centrales y los países de la periferia, tecnológica y científicamente dependientes. Una ilustración privilegiada de la mirada desde los centros de poder acerca del lugar del conocimiento en la sociedad actual, la encontramos en la siguiente cita, extraída de un documento elaborado para el Banco Mundial por Daly, J (1998): “...(se evidencia) un creciente énfasis en el desarrollo internacional basado sobre el conocimiento. El conocimiento se crea y disemina hoy más rápidamente que nunca; la vida del conocimiento es más breve hoy que nunca [...] El conocimiento científico y profesional-tecnológico se ha transformado en el núcleo del desarrollo basado en el conocimiento, y debe dar forma a cada elemento de la sociedad". Esta revalorización se acepta como una verdad indiscutible, sustentada por el pensamiento y la imagen del modelo dominante; se convierte así, en un dato dado y «objetivo», indiscutible, a partir del cual se analiza, se evalúa y se planifica el sistema de educación universitaria y sus conexiones con la sociedad y sus instituciones en gran parte del mundo.

Un argumento central para sostener la falacia de esta consideración acerca del conocimiento, es que para los análisis hegemónicos, se acepta que el conocimiento es uno de los bienes que más ha caracterizado el proceso de globalización vigente a través de las diferencias que se establecen en las nuevas "sociedades del conocimiento" y que explican mediante este artilugio discursivo la 
diferencia entre países. Esta tensión entre globalización del conocimiento y su adecuación a las necesidades locales es, sin duda, otro de los grandes temas de la Universidad argentina en el momento actual. El conocimiento se obtiene en la Universidad a través de prácticas reguladas por la autonomía universitaria y la libertad académica y esto es lo que lo hace tan valioso para la sociedad. Si ellas se subordinan a intereses inmediatistas y pragmáticos, cambian las prácticas y su contexto; el conocimiento así obtenido posee un valor muchas veces limintado sino devaluado- para la sociedad. Es desde esta perspectiva que estamos discutiendo la operación sociologicamente impertinente que implica el énfasis exigencia de mayor en la pertinencia social de la universidad.

Partimos, entonces, de un cuestionamiento a los dualismos que inauguran los abordajes de la noción de pertinencia que suponen que Sociedad y Universidad son objetos aislables, y cuya desconexión - para nosotros imposible - redundaría en Impertinencia. Interpretamos que los consensos actuales acerca de la necesidad de una mayor pertinencia de la Universidad no tienden a vincular aquellas partes que se encuentran desencontradas, sino a redefinir cualitativamente los vínculos entre Universidad y el resto de las instituciones y actores sociales de modos distintos a los que han predominado en las tradiciones universitarias recientes. Una redefinición de vínculos y relaciones que -desde nuestro punto de vista- principalmente se orienta a modificar "función" y "misión" del modelo de Universidad moderna trastocando profundamente varios de los aspectos que, dada su especificidad, distinguen a esta institución social del resto. (NAIDORF; HORN; GIORDANA, 2007) ${ }^{9}$.

En muchas universidades era notable ya a mediados de los 90 que, de hecho, se estaba tendiendo a compartir el «clima académico» tradicional con un cierto «clima empresarial», tanto en lo relativo al modelo de gestión administrativa como en lo que se refería a la organización de las actividades tradicionales de la Universidad, docencia e investigación. Se soslaya el principio de apertura a la

9 Algunas de estas afirmaciones son resultados preliminares de la investigación en curso denominada "Universidad y Sociedad: un abordaje a través de las tensiones y complejidades de la noción de pertinencia" desarrollada en el Instituto Gino Germani de la Facultad de Ciencias Sociales de la Universidad de Buenos. PICT 25508 bajo la dirección del Dr. Francisco Naishtat. 
sociedad del nuevo conocimiento y se lo protege y «privatiza» mediante la adquisición de patentes, licencias y similares medios de defensa de la propiedad privada del conocimiento. Asimismo, los académicos se ven obligados a transformar su tarea e incorporar una mirada empresarial que les permita desarrollar una estrategia en la búsqueda de fondos seguros para sus investigaciones, subvirtiendo así los procesos de investigación científica y otorgándole menor peso a la docencia de grado y posgrado.

En este sentido, cabe mencionar algunas nuevas líneas de política científica y tecnológica destinada a incidir tanto sobre la capacidad de generación de conocimiento de las universidades e institutos de investigación, como de su transferencia.

En 1993 se creó la Secretaría de Ciencia y Tecnología (SECyT). En 1996, a partir del Decreto 660/96, se dispuso el pase de la SECyT al Ministerio de Cultura y Educación, y se emprendió la tarea de la reorganización del sector de Ciencia y Técnica estableciendo dos líneas de trabajo: las cuestiones de mediano y largo plazo, y las exigencias del corto plazo. Entre estas últimas se encuentran las directivas emergentes de los decretos del Poder Ejecutivo Nacional 660/96 y 747/96 referidas a la reorganización de la propia SECyT y del Consejo Nacional de Investigaciones Científicas y Técnicas (CONICET).

Como parte de la reestructuración del Ministerio de Cultura y Educación, por Decreto 1274/96 se aprobó la nueva estructura de la SECyT. Los cambios fueron muy significativos: la SECyT se transformó en el organismo de formulación de la política científica y tecnológica; como tal, tenía la función de elaborar el Plan Nacional Plurianual de Ciencia y Tecnología y el presupuesto del Estado para el sector. Como espacio institucional para la participación de las provincias en la formulación, coordinación y seguimiento de políticas federales de ciencia y tecnología se creó en este ámbito, el Consejo Federal de Ciencia y Tecnología (COFECyT). A partir de esta reestructuración y de acuerdo con el Decreto 1273/96 se creó el Gabinete Científico-Tecnológico (GACTEC) en el ámbito de la Jefatura del Gabinete de Ministros, para la coordinación de las diversas áreas de conducción de actividades científicas y tecnológicas que se llevaron a cabo en distintos ámbitos del Poder Ejecutivo Nacional. Se instituyeron además las Direcciones Nacionales de Planificación y Evaluación, y de Coordinación Institucional, con el objetivo de elaborar los planes y programas en forma participativa y concertada. 
También se creó la Agencia Nacional de Promoción Científica y Tecnológica (ANPCyT), con recursos propios y otros provenientes de créditos de organismos internacionales como el Banco Interamericano de Desarrollo, para otorgar subsidios a la investigación y a la transferencia tecnológica a través de partidas destinadas al Fondo para la Investigación Científica y Tecnológica (FONCyT) y al Fondo Tecnológico Argentino (FONTAR).

De este modo, la SECyT se constituyó en el organismo encargado de la elaboración e implementación de las políticas públicas en materia de ciencia y tecnología, mientras que el CONICET quedó a cargo de llevar a cabo esas políticas de investigación y desarrollo mediante unidades ejecutoras, y de mantener la Carrera del Investigador Científico y Tecnológico, la Carrera del Personal de Apoyo y los Programas de Becas. Por medio del Decreto 1207/96 del Poder Ejecutivo Nacional, se aprobó su nueva estructura organizativa, dependiente de la Secretaría de Ciencia y Tecnología del Ministerio de Cultura y Educación y se definieron sus objetivos y acciones.

Esta reorganización del CONICET y la redefinición del papel de la Secretaría de Ciencia y Tecnología, siguieron los modos de organización de los sistemas de ciencia y tecnología de los países desarrollados y de industrialización reciente, que consideran conveniente la separación institucional de las funciones de: i) formulación de políticas, ii) promoción de actividades científico-tecnológicas, iii) ejecución de actividades científicas y tecnológicas. Este enfoque sostiene que cuando los órganos de promoción están extremadamente subordinados al ámbito de las decisiones políticas, se tiende a descuidar las actividades de investigación básica, y cuando los organismos de promoción cuentan con unidades de ejecución (institutos e investigadores), tiende a perderse la objetividad, desatendiéndose el conjunto de los restantes organismos ejecutores del sistema.

Con el cambio de gestión de gobierno nacional en 1999, la nueva Secretaría de Tecnología, Ciencia e Innovación Productiva (SeTCeIP) cambió nuevamente de jurisdicción y pasó a depender de la Presidencia de la Nación (Decreto 20/99) con responsabilidades en la formulación de políticas, planes, programas, medidas e instrumentos para el establecimiento, puesta en marcha y funcionamiento de un Sistema Tecnológico y Científico Nacional. A comienzos del año 2000 todos los bloques parlamentarios acordaron tratar el Sistema de Tecnología, 
Ciencia e Innovación Productiva como una cuestión de estado. El pacto dio como resultado la Ley 25467 Marco de Ciencia y Tecnología, aprobada en el Congreso de la Nación en 2001, y el Plan Nacional de Ciencia y Tecnología.

La Ley de Ciencia, Tecnología e Innovación, del año 2001, buscó recuperar la responsabilidad primaria del Estado en la organización, gestión y evaluación de las prioridades nacionales para el campo. La misma constituye un intento, aún no ejecutado, de legislar en función de un Sistema Nacional de Ciencia, Tecnología e Innovación en el que las universidades juegan un rol central en tanto generadoras de conocimiento básico, aplicado, y de desarrollos transferibles al sector social y productivo.

En el año 2003 la Resolución No 452/03 del CONICET, denominada "Investigadores en empresas", señala que "las experiencias internacionales reafirman la importancia de realizar los esfuerzos continuos para promover una efectiva articulación entre el sector científico tecnológico y el sector productivo. La vinculación de los centros de excelencia y de los investigadores con las empresas constituyen un factor clave en la transferencia de conocimientos y la valorización de la investigación pública, por lo que el Consejo está convencido de que una cooperación exitosa entre ambos sectores contribuirá en el beneficio del conjunto de la economía del país, al promover la incorporación de valor agregado y generar empleos en mayor número y calificación".

Las universidades han debido aumentar su autofinanciamiento a través de la apuesta a recursos propios para cuya obtención vienen incrementando la captación de fondos extrapresupuestarios a través de convenios de asistencia técnica, pasantías, etc. De este modo, quedaron sometidas a la exigencia de tener que competir por subsidios que en décadas anteriores les garantizaba el Estado, y a ocupar un lugar de mayor protagonismo por sus propias capacidades de administración y gestión institucional.

En relación con la aparición de estos nuevos dispositivos que generan orientaciones en la investigación académica -también nuevasel Consejo Interuniversitario Nacional advirtió que el incremento de la participación del financiamiento privado -fijada su dirección por el Mercado- puede incidir al dar prioridad a ciertas líneas de inves- 
tigación aplicada, que tienen como objetivo resolver problemas específicos de la producción, abandonando aquellas que se considera no darán mayores ganancias ${ }^{10}$.

Estas medidas han producido reacciones heterogéneas entre sus receptores. Una de las críticas fundamentalmente, es la de la precariedad que se percibe en la continuidad de algunos de esos programas que dependen por entero, o casi enteramente, de dinero provisto por los organismos internacionales de financiamiento.

Es importante señalar la plasticidad desarrollada por las universidades, que centran sus esfuerzos de transferencia y de consultoría en el sector terciario de las economías nacionales, provinciales o locales. De esta manera se conectan con el entorno y buscan allí «mercado» para obtener algún rédito económico de sus habilidades y potencialidades.

Por otra parte, la multiplicidad disciplinaria, la pluralidad de demandas e intereses - internos y externos - de la comunidad científica, la diversidad de fuentes de financiación por las cuales se compite, la variedad histórica respecto a la trayectoria de investigadores y grupos de investigación, así como también respecto a las experiencias institucionales, modeló en Argentina un perfil de la actividad investigativa más bien de adaptación, que dé respuesta a una planificación y a un conjunto coherente de decisiones.

\section{iv) La resignificación de la extensión universitaria}

En la década del 60, la universidad argentina se caracterizó por la centralidad de la extensión universitaria como la principal dimensión de su función social, proyectándose para intervenir en los problemas de aquellos estamentos sociales con menor capacidad para incidir en las estructuras de poder.

Durante la década de los 90 se produjo una fuerte caída en la cantidad de proyectos presentados, al tiempo que se evidenciaba un fuerte aumento en los ingresos de recursos propios a partir de la venta de servicios en muchos casos ofrecidos desde las mismas dependencias que realizaban acciones de extensión universitaria. De este modo, el nuevo modelo de universidad estaba planteando un cambio en los propósitos y en los destinatarios de la relación entre

10 Ver Coraggio; Vispo (2001). 
Universidad y Sociedad, configurando un nuevo patrón de universidad, despojada de sus perfiles sociales, para subsumirla a un tipo de vinculación con el sector productivo-empresarial, con el fin de disponer de ingresos para su autofinanciamiento progresivo. Al respecto, algunos investigadores afirman que

la interacción entre la Universidad y el sector productivo está hoy experimentando un cambio importante. En los países industrializados y recientemente también en América Latina, se fue redefiniendo la tradicional función de extensión universitaria, adquiriendo mayor valor la transferencia de aquellos conocimientos de utilidad para el desarrollo económico y tecnológico del país que, simultáneamente, pudieran reportar un beneficio económico para la universidad. (GARCÍA DE FANELLI, 1993) ${ }^{11}$.

Desde otras perspectivas se aboga en la actualidad por la recuperación de los principios políticos y de los criterios que, explícita o implícitamente, daban fundamento a la organización de las actividades de extensión desde sus orígenes. La concepción de la función social de la Universidad, en este marco, adquiere un sentido específico al considerarse que la práctica universitaria debe desarrollarse en contacto permanente con el medio social y especialmente con los sectores populares. Esta postura debate o confronta con los modelos de extensión universitaria que optan por la idea de que las instituciones de educación superior deben desarrollar una política de recuperación de costos y que, por lo tanto, la relación de la Universidad con organizaciones externas debe consistir en la venta de servicios profesionales que permitan incorporar recursos para el autofinanciamiento (BRUSILOVSKY, 2000). Es decir que mientras algunas universidades incluyen las actividades de vinculación con empresas en el área de extensión universitaria, (por mencionar sólo algunas, las universidades de San Juan, San Luis, del Sur, de Jujuy, de La Pampa), en otras se llevan a cabo desde las Secretarías de Ciencia y Técnica (como en la Universidad Nacional de la Patagonia Austral, Universidad de La Rioja, de Misiones, entre otras).

El contexto nacional que aquí se ha resumido, muestra el intento de sistematizar las principales acciones de política científica implementadas en Argentina a lo largo de los últimos veinte años, en

11 Una ampliación de este debate puede encontrarse en Iazzetta (2001). 
relación con la promoción de la vinculación Universidad - Empresa. Mediante medidas más o menos específicamente destinadas a cumplir con este objetivo, las distintas acciones de reformas universitaria en el marco del complejo científico - tecnológico (OTEIZA, 1992), han buscado generar un cambio profundo en la cultura académica de la Universidad pública, y en sus formas y objetivos en tanto ámbito de producción de conocimiento. Asimismo, la sola comparación de las partidas presupuestarias para investigación y extensión proporciona un dato ilustrativo de la asimetría entre ambos procesos.

Por otra parte, el ya mencionado "Programa Nacional de Incentivo a Docentes Investigadores" ha sido y sigue siendo uno de los instrumentos que mayor impacto tuvo sobre las prácticas docentes en detrimento de la función de extensión ${ }^{12}$.

Con la categorización y evaluación se impone un sistema paralelo al de los concursos docentes. Para no quedar excluido, todo investigador debe acreditar tareas de docencia y todo docente debe investigar. Aclaremos que el dinero aportado como una suerte de «sobresueldo en negro» constituye, muchas veces, una parte sustancial de lo que los docentes perciben como remuneración (VOMMARO, 2003).

Además, la asimetría entre investigación y extensión se profundizó con otros dos programas: el de "Premio Estímulo a la Producción Científica" y el de "Formación de Recursos Humanos". Esto no significa que se deba desalentar la vigencia de dichos programas, sino ampliar su aplicación a las prácticas extensionistas. En lo formativo, la disparidad en algunas universidades nacionales puede observarse en el tipo de becas y la proyección en la formación de los sujetos involucrados, ya que en investigación los graduados pueden acceder a becas de maestría y doctorado, con una duración de dos años por período -informes mediante- y dedicados exclusivamente a esta actividad. Las de extensión, en cambio, se asignan generalmente a alumnos antes que a graduados y con escasas posibilidades de renovación. También aquí adquiere relevancia el aspecto económico, ya que las becas de investigación no solo son de un monto significativamente mayor que las de extensión, sino que incluyen la posibilidad

12 Consiste, desde su primera implementación en 1993, en un plus salarial que se paga en tres cuotas anuales fijadas según una categorización a la que fueron sometidos los docentes previamente. En un principio, se fijaron cuatro categorías (de la A a la D) en las que eran clasificados según el desarrollo de sus tareas de docencia e investigación. En el año 2004 se realizó la última recategorización de los docentes investigadores que estableció jerarquías entre la 1 y la 5. 
de contar con la obra social de la universidad. Por otro lado, a través de las "remuneraciones adicionales" a quienes desarrollan servicios a terceros y/o transferencias, se fue configurando e imponiendo otro aspecto del nuevo arquetipo de universidad: la reorientación de la actividad de los docentes que -en muchos casos-apremiados por salarios congelados fueron abandonando la extensión debido a que las otras actividades resultan económicamente más atractivas. Se obliga a los programas y servicios de las universidades a adquirir una dirección específica que asegure la colocación de sus productos en el mercado.

El elemento más importante para enriquecer el debate acerca de las cuestiones pendientes referidas a la extensión universitaria, sigue siendo el de una "vocación social" que se aparte de la idea de asistencia y/o servicio. Habría que recordar que conforme a los principios del "Manifiesto Liminar de la Reforma Universitaria de 1918", las conquistas científicas y culturales, aunque hayan sido producto del trabajo intramuros, constituyen patrimonio común de todos los miembros de la sociedad.

\section{REFLEXIONES FINALES}

Como señala Wallerestein, la relación entre la Universidad y el Estado por un lado, y la sociedad civil, por otro lado, siempre ha sido difícil, ambigua, y llena de tensiones e incluso de conflictos. No obstante, la Universidad juega un rol central en la vida de los pueblos y un pueblo no podría jamás tener una existencia sana sin una universidad vigorosa y crítica; debe ser un centro de pensamiento crítico, como "una especie de poder intelectual que la sociedad necesita para que la ayude a reflexionar, comprender y actuar" (INFORME DELORS, 1989).

Sin embargo, hoy la Universidad se encuentra en la encrucijada de la exigencia a transformar su relación con el medio sociocultural inmediato, especialmente el relacionado a la dimensión de la economía y la producción; exigencias que se expresan en la necesidad de una adecuación a éste último, con demandas de mayores niveles de eficiencia y productividad. No puede quedar al margen de este análisis, que el discurso hegemónico de las últimas décadas ha instalado una racionalidad economicista para pensar los problemas de educación, amparada en una necesidad de adecuación del sistema educativo a las 
necesidades del mercado, exponiendo a las instituciones a procesos de acreditación académica para obtener fondos según criterios extrínsecos. Estas exigencias no parecen privilegiar la ampliación del acceso y permanencia de postulantes de estratos socioeconómicos desfavorecidos. La baja cobertura del crédito educativo y los sistemas de becas que privilegian los estudios de postgrado, han tenido como resultado un impacto bajo en la expansión de las oportunidades de acceso a la educación superior.

La crisis de autonomía planteada en Argentina resulta evidente al observar las medidas gubernamentales implementadas en la modalidad de programas de incentivos y de fomento diferencial, tendientes al logro de esa mayor eficiencia y productividad. La implementación de estas reformas necesitó la construcción por parte de las autoridades de un consenso entre diferentes grupos dentro de la comunidad universitaria. Sin embargo, es posible repensar la cultura institucional de las universidades públicas, articulando nuestros intereses con los de la sociedad, y exigiendo al Estado que a través de los recursos presupuestarios -necesarios y suficientes-, se garantice el financiamiento de la ciencia, la educación y la cultura, puesto que estas actividades, así como las de extensión e investigación, no son fines en sí mismos, sino la llave para garantizar una mejor calidad de vida para el pueblo y la posibilidad de que, a través de ellas, la universidad pueda seguir formando parte del proceso de transformación social. Afirmar que la educación superior es un servicio público, significa realzar el papel de la Universidad en la construcción y desarrollo social y humano, para lo cual el Estado debe establecer políticas que promuevan, regulen y financien la educación superior; tal como están planteadas las relaciones de poder sólo el Estado está en condiciones de coordinar adecuadamente el uso de los recursos y de priorizar y financiar áreas no rentables en lo inmediato. En este sentido, la forma de preservar la integridad de la Universidad radica en el compromiso de los miembros de la comunidad académica por involucrarse en la dirección de la institución, garantizando y fortaleciendo su función académica. 


\section{REFERÊNCIAS}

BANCO MUNDIAL. Departamento de Educación y Política Social. La enseñanza superior. Las lecciones derivadas de la experiencia, 1995.

BANCO MUNDIAL. Informe sobre el desarrollo mundial 1998/99. Washington D.C., 1999.

BISANG, R. Las actividades de investigación en las Universidades Nacionales Secretaría de Políticas Universitarias. Ministerio de Cultura. Serie de Estudios y Propuestas. Buenos Aires, 1995.

BRUSILOVSKY, Silvia D. Extensión universitaria y educación popular. Libros del Rojas. Buenos Aires: Eudeba; Universidad de Buenos Aires, 2000.

BUENOS AIRES. Secretaría de Políticas Universitarias. Programa de vinculación tecnológica en las universidade. Primeras jornadas nacionales de vinculación tecnológica en las universidades. Buenos Aires: Ministerio de Cultura y Educación, 1995.

CASANOVA Cardiel, H. Universidad: idea y racionalidad contemporánea. En: DÍAZ BARRIGA, A.; PACHECO MÉNDEZ, T. (Coords.). Universidad: institucionalización académica y evaluación. México: CESU-UNAM, 1997.

CORAGgio, J. L.; VISPO, A. Contribución al estudio del sistema universitario Argentino. Buenos Aires: CIN; Miño y Dávila, 2001.

CHIROLEU, A. El ingreso a la universidad. Las experiencias de Argentina y Brasil. Rosario: UNR, 1999.

DALY, J. The World Bank Foundations in Science and Technology for Development: a background paper for a panel discussion. [s.1.]: [s.n.], 1998.

GARCÍA DE FANELLI, A. La articulación de la UBA con el sector productivo: la experiencia reciente. Buenos Aires: [s.n.], 1993. Doc. CEDES/96

IRIARTE, Alicia (Comp.). La universidad pública argentina ¿crisis o encrucijada?. Buenos Aires, Argentina: Proyecto Editorial, 2004.

IAZZETTA, O. La recreación de la dimensión pública de la universidad. Pensamiento Universitario, Buenos Aires, n. 9, 2001.

LLOMOVATTE, $S$. et al. Vinculación de la universidad con la empresa: debates, políticas y estudios de casos. Buenos Aires: Editorial Miño y Dávila, 2006. 
LLOMOVATTE, S. Universidad y empresa en Canadá. La universidad ahora, Buenos Aires, 1999.

MANIFIESTO Liminar de la Reforma Universitaria de 1918.

MARTINETTO, Alejandra. Crisis universitaria y posibilidades de construcción de un nuevo modelo hegemónico.En: ENCUENTRO NACIONAL, 5; LATINOAMERICANO LA UNIVERSIDAD COMO OBJETO DE INVESTIGACIÓN, 2, 2007, Tandil, Provincia de Buenos Aires, Argentina.

NAIDORF, Judith. Privatización del conocimiento público en universidades públicas. En: GENTILI, Pablo; LEVY, Bettina (Comp.). Espacio público y privatización del conocimiento. Estudios sobre políticas universitarias en América Latina. Buenos Aires: CLACSO, oct. 2005.

NAIDORF, J.; HORN, M.; GIORDANA, P. Las tensiones y complejidades de la noción de pertinencia. En: ENCUENTRO NACIONAL, 5; LATINOAMERICANO LA UNIVERSIDAD COMO OBJETO DE INVESTIGACIÓN, 2, 2007, Tandil, Provincia de Buenos Aires, Argentina.

SCHUGURENSKY, D.; NAIDORF, J. Parceria universidade-empresa e mudancas na cultura academica: análise comparativa dos casos da Argentina e Canadá. Educacao \& Sociedade, Campinas, SP, v. 25, n. 88, 2004. Especial. Universidade: reforma e/ou rendição ao mercado?

PORTER, Luis. Globalización en la producción del conocimiento: políticas internacionales, su influencia en las políticas nacionales y su efecto en las universidades públicas mexicanas. In: CONFERENCIA CONGRESO DE INVESTIGACIÓN UAM, 2001, Xochimilico, México.

PRATT, Linda R. A new face for the profession. Academe. Bulletin of the American Association of University Professors, Sept., 1994.

OTEIZA, E. La política de investigación científica y tecnológica argentina. Buenos Aires: Centro Editor de América Latina, 1992.

TENTI, E. Universidad y empresa. Buenos Aires, Argentina: Editorial Miño y Dávila; Ciepp, 1993.

VOMMARO, Pablo. Reforma y ajuste en la Universidad de Buenos Aires. Revista Memoria, Chile, n. 173, 2003.

WALLERSTEIN, I. Retos para la universidad en el siglo XXI. Investigaciones Sociales, Lima, año 8, n. 13, 2004.

WINCHESTER, I. The concept of university autonomy. An anachronism? OISE, Toronto, Canada, 1985. 